\title{
Development of Au-GCIB Dynamic SIMS and Cluster Size Filtering System
}

\author{
M. Nojima ${ }^{1,2}$, M. Suzuki ${ }^{2}$, T. Adachi ${ }^{2}$, S. Hotta ${ }^{3}$, M. Fujii ${ }^{4}$, T. Seki ${ }^{5}$ and J. Matsuo ${ }^{4}$ \\ ${ }^{1}$ Tokyo University of Science, RIST, 2641 Yamazaki, Noda, JP-278-8510 Chiba \\ 2 Tokyo University of Science, Faculty of Sci. and Tech., 2641 Yamazaki, Noda, JP-278-8510 Chiba \\ ${ }^{3}$ Office Tandem. LCC., 2-16-8 Nishi-cho Kokubunji-shi, JP185-0035 Tokyo \\ ${ }^{4}$ Quantum Science and Engineering Center, Kyoto University, Gokasho, Uji, JP-611-0011 Kyoto \\ ${ }^{5}$ Department of Nuclear Engineering, Kyoto University, Gokasho, Uji, JP-611-0011 Kyoto
}

Recently, Ar- gas cluster ion beam (GCIB) is discussing as a most promising primary ion beam for organic SIMS (i). Prof. Matsuo's group firstly developed a linear type Ar-GCIB TOF-SFMS system (ii). Prof. Mochiji's group developed a size-selected Ar-GCIB as a projectile with a linear type TOF-SFMS system (iii,iv). Also Prof. Matsuo's group has developed an orthogonal acceleration type Ar-GCIB TOF-SIMS system, which is modified for direct primary ion beam bombardment by chopping secondary ion beam (v,vi). In this study, we have introduced Ar- GCIB to a dynamic SIMS system: FMS-4f.

IMS-4f is strongly modified for inorganic analysis introducing duo-plasmatron and Cs microbeam ion source. The apparatuses are wide spreading all over the world, namely in Japan, USA and Europe. The systems are simply driven and easy to convert. There is a report that $\mathrm{C}_{60}{ }^{+}$ion source was introduced to FMS-4f (vii). The advantages using IMS-4f as a test-bed for Ar-GCIB applications are probabilities to demonstrate new potentials for big seller CAMECA IMS series. Also considerable applications are bio-imaging using the projection secondary ion image. The projection image resolution is $0.5 \mu \mathrm{m}$ without scanning the primary ion beam in an official value.

In this study, we have obtained mass spectrums of organic samples by developed Ar- GCIB dynamic SIMS system. Figure 1 indicates mass spectrums of arginine irradiated by Ar-GCIB (a), polystyrene (PS) irradiated by $\mathrm{O}_{2}{ }^{+}$beam (b) and Ar-GCIB (c). All the organic samples are spin coated on Si wafer.

On the spectrum (a), main peak of protonated arginine and other fragment peaks are clearly observed. The mass spectrum is similar to obtained by Ar-GCIB TOF-SFMS (viii). Typical organic mass peaks originated PS (tropylium ion and depolymerized cations) are clearly observed on mass spectrum (b). The mass spectrum represents similar pattern compared to the one of thermal-assisted atmospheric pressure glow discharge mass spectrometry and the fragmentation mechanism is described in the article (ix). On $\mathrm{O} 2^{+}$beam radiation (c), typical organic mass peaks are disappeared and mass peaks are shifted to low mass positions. Acceleration voltage of $\mathrm{O}_{2}{ }^{+}$beam was $3 \mathrm{keV}$. On the higher acceleration voltage condition on $\mathrm{O}_{2}^{+}$beam, low mass peaks are also disappeared. It can be said that soft sputtering conditions are keeping on Ar- GCIB dynamic SIMS system.

In our developed system, Ar-GCIB is filtered only by a permanent magnet. It is anticipated that only monomer Ar ions are mass filtering. At least, we cannot control the size of clusters. It is reported that fragmentation of secondary ions can be controlled by selecting the size of clusters (iii). So we are now developing a new cluster size filtering system. The system is based on physical behavior of ions in rotating electric field. In rotating electric field, ions draw cycloidal trajectories. The trajectories are different according to the cluster sizes. The cluster size filtering system introduced two rotating electric fields reversing each phase. The cluster sizes are filtered in each rotating electric field. The rotating electric fields are generated by two sets of octupole (Fig. 2). The features of cluster size filtering system are listed below.

1. Quite a wide mass range: 1-40,000 AMU 
2. Compact packaging: the size is almost same as a quadrupole mass analyzer

3. Fine for ion focusing: without chopping plate, symmetrically equal to ion trajectory

The cluster size filtering system will be a powerful tool for Ar- GCIB dynamic SIMS system.
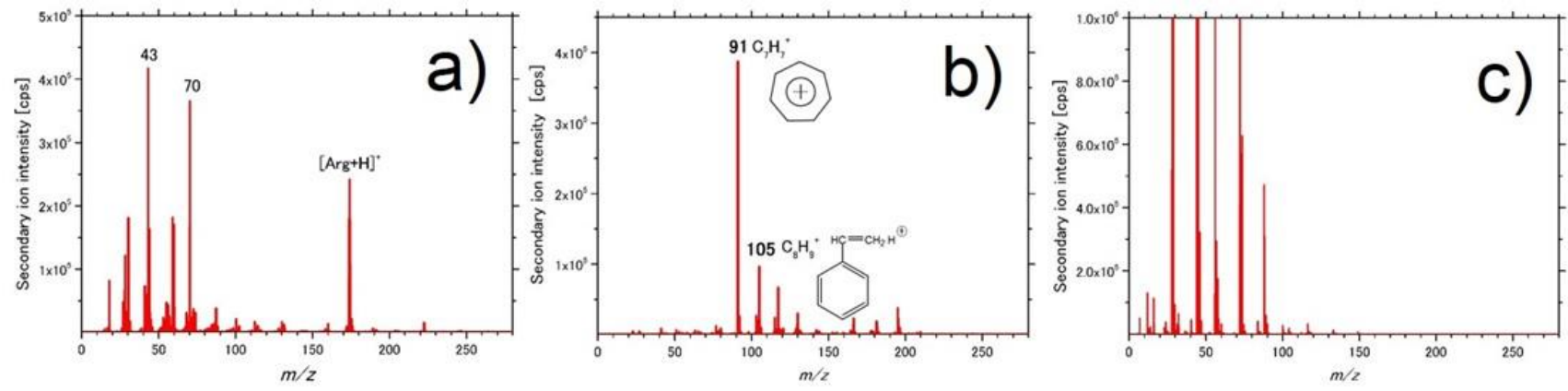

Arginine irradiated by Ar-GCIB (a), polystyrene (PS) irradiated by $\mathbf{O 2}$ beam (b) and Ar-GCIB (c)

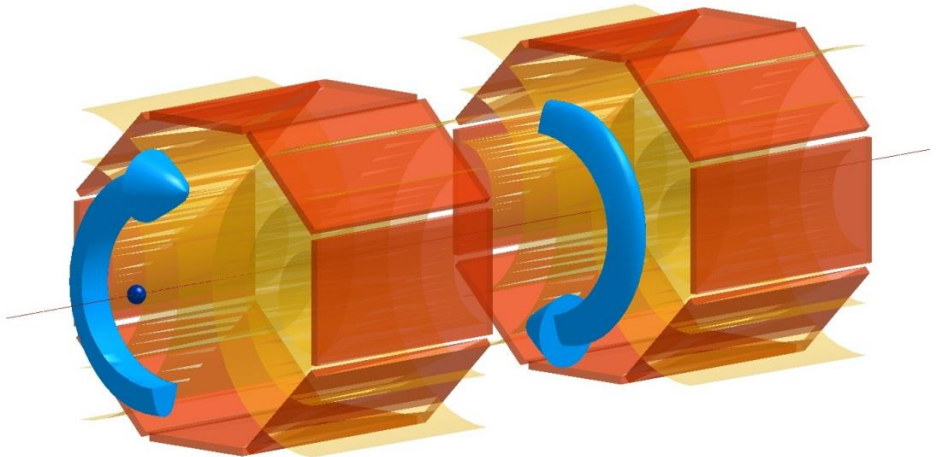

Fig. 2 A new cluster size filtering system introducing two rotating electric fields reversing each phase.

\section{References}

(i) J. Matsuo, S. Ninomiya, Y. Nakata, Yoshiro Hondab, K. Ichiki, T. Seki and T. Aoki, Applied Surface Science Vol. 255, 2008,1235-1238

(ii) S. Ninomiya, Y. Nakata, K. Ichiki, T. Seki, T. Aoki and J. Matsuo, Nuclear Instruments and Methods in Physics Research Section B, Vol. 256, Issue 1, 2007, 493-496

(iii) K. Moritani, M. Hashinokuchi, J. Nakagawa, T. Kashiwagi, N. Toyoda, and K. Mochiji, Applied Surface Science

Vol. 255, 2008, 948-950

(iv) K. Moritani, G. Mukai, M. Hashinokuchi, and K. Mochiji, Applied Physics Express, Vol. 2, 2009, 046001

(v) H. Gnaser, M. Fujii, S. Nakagawa, T. Seki, T. Aoki and J. Matsuo, Rapid Commun. Mass Spectrom. , Vol. 27, 2013, 1490-1496

(vi) K. Ichiki, J. Tamura, T. Seki, T. Aoki and J. Matsuo, Surface and Interface Analysis, Vol. 45, 2013, $522-524$

(vii) G. Gillen, J. Batteas, C. Michaels, P.Chi, J. Small, E. Windsor, A. Fahey, J. Verkouteren and K. J. Kim, Applied

Surface Science, Vol. 252, 2006, 6521-6525

(viii) J. Matsuo, K. Ichiki, Y. Yamamoto, T. Seki and T. Aoki, Surface and Interface Analysis, Vol. 44, Issue 6, 2012, 729-731

(ix) N. Zhang, Y. Zhou, C. Zhen, Y. Li, C. Xiong, J. Wang, H. Li and Z. Nie, Analyst, Vol. 137, 2012, $5051-5056$ 